\title{
Studies on the Role of Fibrinogen on Lipid Deposition into the Aorta
}

\author{
Mototaka Murakami, M.D., Hiroshi Sekrmoto, M.D., \\ Masuzo Tatsuguchi, M.D., Yoshiaki Yasuda, M.D., \\ Sumio Masuda, M.D., Kazuyuki Matsumoto, M.D., \\ Toshio Shinagawa, M.D., Akira Genda, M.D., \\ Takekuni Ikeshima, M.D., Hidekazu Ozaki, M.D., \\ Takio Yamada, M.D., and Masao Murakami, M.D.
}

The purpose of this report was to determine whether the presence of fibrinogen has an effect on the deposition of $\beta$-lipoprotein into the aorta. Chromatography with citrated ethanol saline system displayed lipids at the origin, only when chromatograms of samples of acid polysaccharides plus plasma were run on filter papers. They were absent from preparations of acid polysaccharides and serum. The results obtained with the fluorescentantibody technique indicated that accumulations of $\beta$-lipoprotein in the intima were associated with the presence of fibrin. It is suggested that fibrinogen enhances the deposition of lipid complexes from abnormally lipaemic sera.

$I^{1}$

$T$ is indicated in the work of various authors that at least a part of the lipids found in the arterial wall is synthesized by that organ in situ. On the other hand, it has been demonstrated that, under experimental conditions at least, the earliest stages of lipid deposition in vascular intima could be initiated primarily by certain low density cholesterol containing lipoprotein complexes without the participation of cells or antecedent fibrinous encrustation.

Our own studies have shown that arteriosclerotic lesions are accompanied by profound alterations in the mucopolysaccharides of rabbit and human aortae. In our previous report, ${ }^{1,2)}$ extracts of human aortae were shown to contain materials with immunological properties consistent with those of $\beta$-lipoprotein or fibrin. Furthermore, we had found that $\beta$-lipoprotein or fibrin was present in greater concentration in severely sclerotic aortae than in mildly sclerotic or uninvolved ones. On the other hand, Bernfeld et al. ${ }^{3}$ found that $\beta$-lipoprotein and fibrinogen form specific complexes with certain macromolecular polysulphates and these results were demonstrated by us with mucopolysaccharides of human aortae. The data obtained by us suggest that $\beta$-lipoprotein and fibrinogen are deposited in aortae in cxactly the samc way. It would be of considerable interest to determinc whether

From the Second Department of Internal Medicine, School of Medicine, Kanazawa University, Kanazawa. 
the presence of fibrinogen has an effect on the deposition of $\beta$-lipoprotein into the aorta.

\section{Methods and Materials}

\section{Paperchromatography}

We have followed the uni-dimensional descending technique, using Toyo Roshi No. 50 paper and solvent mixture; $0.15 \mathrm{Gm}$. sodium citrate solution, ethanol and $0.9 \%$ sodium chloride solution, $5: 28: 67$ parts by volume.

To study interactions of acid mucopolysaccharides, fibrinogen and $\beta$-lipoprotein, single and equivolume mixture of them were applied.

Samples in application:-

1) Fibrinogen solution: $40 \mathrm{mg}$. of fibrinogen (Armor Pharmaceutical Company) dissolved in $1 \mathrm{ml}$. of citrated $0.9 \%$ sodium chloride solution. saline.

2) Heparin solution: 1,000 units of heparin dissolved in $1 \mathrm{ml}$. of the citrated

3) Sulfated alginic acid solution: One half $\mathrm{mg}$. of sulfated alginic acid dissolved in $1 \mathrm{ml}$. of the citrated saline.

4) Human aorta-acid mucopolysaccharides solution: $3 \mathrm{mg}$. of acid mucopolysaccharides isolated from human aorta were dissolved in $1 \mathrm{ml}$. of the citrated saline.

The isolation of the mucopolysaccharides was made according to the method of Dyrbye and Kirk, ${ }^{4)}$ somewhat modified by us.

Five or 6 hours were sufficient time for the solvent to reach about $3-4 \mathrm{~cm}$. from the bottom of the paper. After this time, the solvent boundary was marked and the paper air dried. It was then fixed for $20 \mathrm{~min}$. with a mixed solution: 10\% formalin and ethanol, 1:4 parts by volume. The papers were dried under diminished pressure to remove the aldehyde and then stained with various methods.

1) Acid mucopolysaccharide stain

The fixed paper strips were transferred into staining racks and stained for 10 min. in staining trays with $0.5 \%$ toluidine blue in $80 \%$ aceton solution; after staining, the strip was washed with $0.1 \%$ acetic acid solution to remove excess dye.

2) The strips were stained for $10 \mathrm{~min}$. with dye solution (bromphenol blue $0.05 \%$, mercuric chloride $1 \mathrm{Gm}$.); after staining, the strips were washed with washing solvent (mercuric chloride $1 \mathrm{Gm} ., 2 \%$ acetic acid $100 \mathrm{ml}$.).

3) Lipoprotein stain

The strips were stained for 30 min. with saturated Sudan Black B $70 \%$ ethanol solution, and then washed several times to remove excess dye with $70 \%$ ethanol.

2. Preparation of antiserum to human fibrin

The antiserum used for conjugation was obtained by immunization of adult rabbits with human fibrin.

The fibrin was prepared by adding $10 \%$ calcium chloride solution to centrifuged human plasma. Two hours after incubation at $37^{\circ} \mathrm{C}$ the resultant clot was separated. This fibrin clot was repeatedly pressed out with glass rod to remove the serum component in normal saline and then ground in glass grinder.

The homogenate was centrifuged 6,000 r.p.m. for $20 \mathrm{~min}$. The precipitate was suspended and washed with normal saline and then centrifuged as above. 
This procedure was repeated 10 times to purify the fibrin. The purified fibrin was redissolved with $0.1 \mathrm{~mol}$. oxalic acid, and their soluble fibrin fraction dialysed against tap water for several hours. The dialysate was centrifuged 6,000 r.p.m. for $20 \mathrm{~min}$. The precipitate was used as antigen with Freund's adjuvant. Two intramuscular injections of $10 \mathrm{mg}$. of the purified ground fibrin were given at a week followed by 8 injections. Two weeks after the terminal injection, antiserum was prepared by treating with barium sulphate to remove prothrombin and any trace of thrombin.

3. Preparation of antiserum to human $\beta$-lipoprotein

$\beta$-Lipoprotein was isolated from human serum by adding the 20 fold volumes of $4 \mathrm{mg}$. per cent dextran sulfate solution. One hour after the mixing of this solution, the mixture was centrifuged 6,000 r.p.m. for $20 \mathrm{~min}$. The precipitate was suspended in the dextran sulfate solution and centrifuged again. This procedure was carried out several times to remove other contaminants. Two intramuscular injections of $20 \mathrm{mg}$. of the isolated $\beta$-lipoprotein were given with Freund's adjuvant at a week followed by 8 injections. Two weeks after the terminal injection, an antiserum was prepared.

\section{Preparation of fluorescein-coupled antiserum}

Globulins were precipitated in the cold from the above crude 2 rabbit sera by half saturated with ammonium sulfate. The precipitate was redissolved in normal saline and the globulin solution was dialysed to remove ammonium sulfate.

These globulins were conjugated with fluorescein isothiocyanate and absorbed with washed blood group $\mathrm{O}$ red cells in order to remove antibodies formed in response to traces of other serum proteins which might have contaminated the antigen. It had a fluorescein/protein ratio of 1:200. The conjugated dye was removed by Sephadex G50 column technique.

\section{Treatment of sections}

The material studied came from the aortae of old men necropsy. The lesions selected were irregular, slightly yellow in colour and minimally elevated. Lesions from the ascending part of the aorta were studied.

A block of fresh tissue was chilled at $-70^{\circ} \mathrm{C}$ in a mixture of acetone and dry ice and stored at $-20^{\circ} \mathrm{C}$ until required.

Sections were cut $2-3 \mu$ thick at $-20^{\circ} \mathrm{C}$ in cryostat. The sections were mounted on aluminised slides and dried. They were treated with buffered saline $(\mathrm{pH} 7.1)$, then flooded with fluorescein-coupled antisera and left in a moist atmosphere at room temperature for $40 \mathrm{~min}$. After staining, they were washed with the above chilled buffered saline in order to remove excess fluorescein-coupled antisera, and mounted in $10 \%$ glycerine buffered saline. They were then examined with a fluorescent microscope.

\section{RESULTS}

1. The chromatographic results

After spotting of the substances on Toyo Roshi No. 50 paper, the chromatography was carried out with citrated ethanol saline system at room tem- 
perature using a descending technique. Under these conditions heparin and fibrinogen migrated with the solvent front, whereas fibrinogen plus heparin and fibrinogen plus sulfated alginic acid left metachromatic compounds stained with toluidine blue at the origin (Fig. 1).

The results of metachromatic staining and lipid staining of human serum plus human aortic mucopolysaccharides were shown in Fig. 2. In both cases no spots could be detected with these stains at the origin.

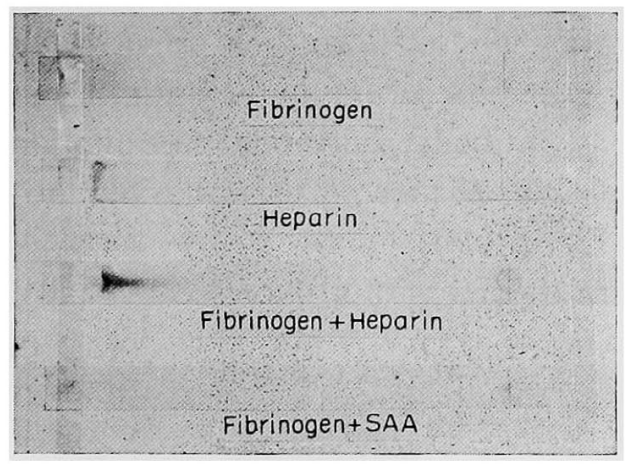

Fig. 1. Descending chromatograms (toluidine blue stain) of fibrinogen, heparin, heparin plus fibrinogen and sulfated alginic acid plus fibrinogen.

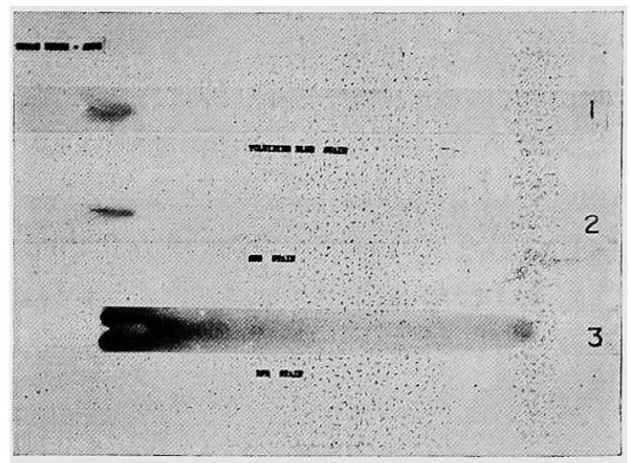

Fig. 2. Descending chromatograms of human serum plus acid mucopolysaccharides prepared from human aortae. 1, Toluidine blue stain; 2, Sudan black B stain; 3, Bromphenol blue stain.

When chromatograms of the mixture of human plasma and human aortic mucopolysaccharides were run on paper, lipids could be detected at the origin, whereas they were absent from the mixture of human serum and human acid mucopolysaccharides. They were identified with Sudan Black B, but they also stained with toluidine blue. There were no other Sudan Black

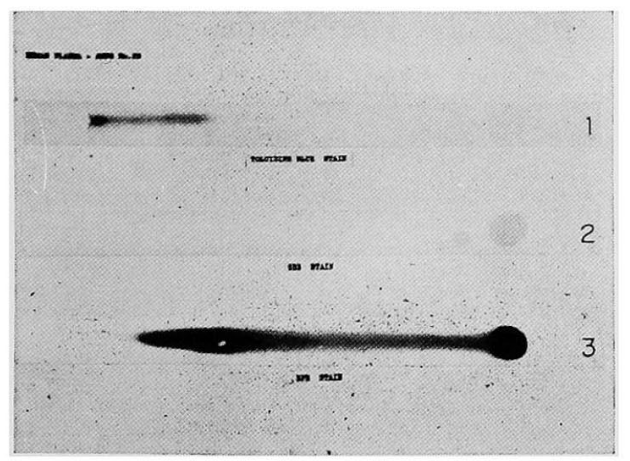

Fig. 3. Descending chromatograms of human plasma plus acid mucopolysaccharides prepared from human aortae. 1, Toluidine blue stain; 2, Sudan black B stain; 3, Bromphenol blue stain.

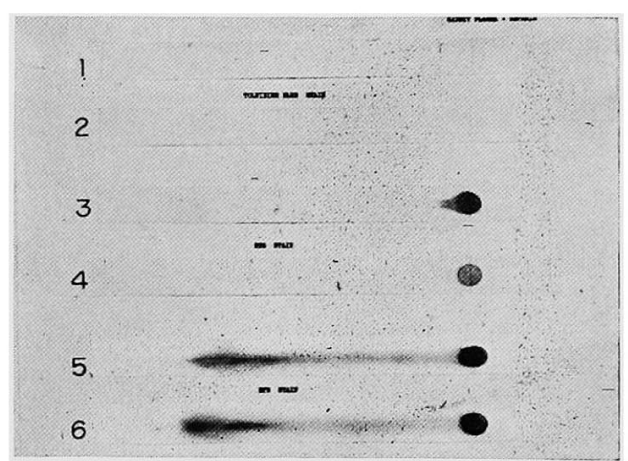

Fig. 4. Descending chromatograms of rabbit plasma plus heparin. 1 and 2, Toluidine blue stain; 3 and 4, Sudan black B stain; 5 and 6, Bromphenol blue stain. 
B staining spots detectable on the chromatograms (Fig. 3).

Fig. 4 shows the results of one experiment with samples of rabbit plasma plus heparin. Staining with Sudan Black B revealed lipids at the origin, which also took up the toluidine blue.

2. The immuno-histochemical results.

The rabbit anti-fibrin serum and rabbit anti- $\beta$-lipoprotein serum were excellent. When plasma was subjected to immunoagar-gel diffusion by the method of Ouchterlony using as an antibody the rabbit anti-fibrin rabbit serum of a high titer, only one precipitation line of fibrinogen origin could be observed (Fig. 5). Furthermore, using human serum as the antigen and rabbit anti-human- $\beta$-lipoprotein serum as antibody, only one precipitation line was noted, indicating that, from the point of view of precipitating antibody, the antiserum was monospecific (Fig. 6).

Frozen sections of human aortae were treated with a fluorescein-coupled

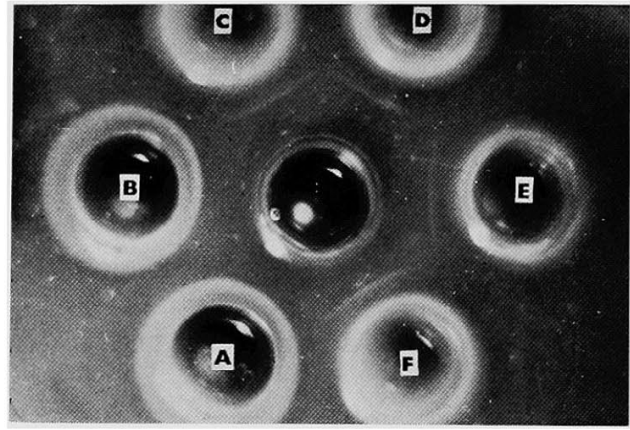

Fig. 5. Central basin, anti-human fibrin serum. Peripheral basins to be tested. The basins, A and B filled with human sera, C, D, E, and F with plasma.

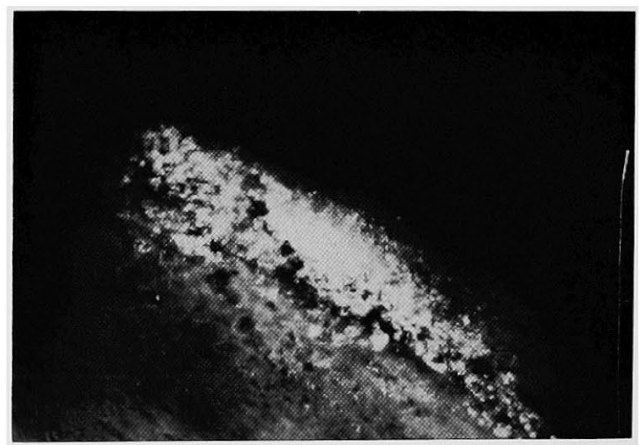

Fig. 7. Section through atheromatous plaque treated with fluorescein-labelled antifibrin serum.

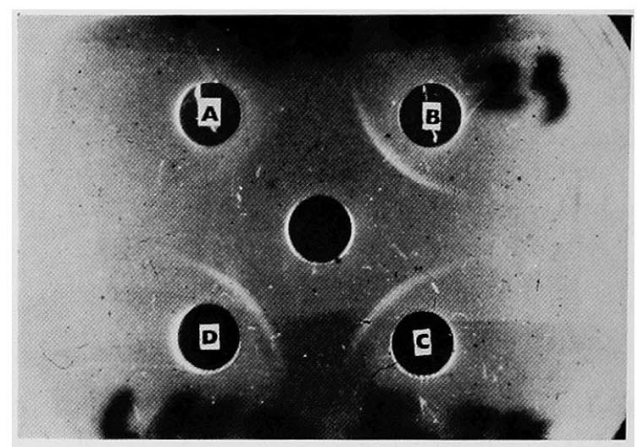

Fig. 6. Central basin, anti-human $\beta$-lipoprotein serum. Peripheral basins to be tested. The basins, A filled with $\beta$ lipoprotein free serum, B, C, and D with $\beta$-lipoprotein rich sera.

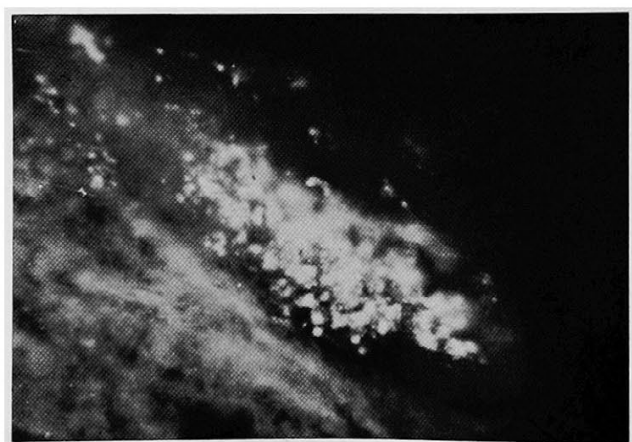

Fig. 8. Section through atheromatous plaque treated with fluoresceinlabelled anti- $\beta$-lipoprotein serum. 
rabbit anti-human-serum to identify fibrin. The thickened intima showed abundant specific yellow green flourescence forming irregular aggregates (Fig. 7). Sections adjacent to those stained with the labelled anti-fibrin serum were then flooded with labelled anti- $\beta$-lipoprotein serum. Specific fluorescence was consistently observed in the thickened intima. It was of great interest that the fluorescein-labelled anti-fibrin serum was concentrated in the same areas as the major collections of the fluorescein-coupled anti- $\beta$-lipoprotein serum (Fig. 8).

\section{Discussion}

In our previous reports, extracts of human aortae were shown to contain materials with immunological properties consistent with fibrin. Recently Woolf and Crawford ${ }^{5,6)}$ have applied the Coons' fluorescent antibody technique to a study of fatty streaks in the human aorta in an attempt to determine whether fibrin incorporation is in fact associated with these early lesions. They consistently found specific fluorescence, indicative of the presence of fibrin. Bernfeld et al. found that certain macromolecular polyanions form specific complexes with only 2 fractions of the plasma proteins, namely $\beta$ lipoproteins and fibrinogen. Our hypothesis is that the accumulated vascular mucoid substances might form similar complexes and that this might be the first stage leading to the deposition of lipid and fibrin. Stewart ${ }^{2)}$ reported that when plasma or plasma-like solutions containing fibrinogen were pulsated against isolated segments of young rabbits' aortae fibrinoid substance was deposited on the intima. Furthermore he noticed that the fibrinoid substance enhanced the deposition of lipid complexes from abnormally lipaemic sera.

Chromatography with citrated ethanol saline system displayed lipids as before at the origin only when chromatograms of samples of acid polysaccharides plus plasma were run on filter papers. They were absent from preparations of acid polysaccharides and serum. The data obtained by us support the view that lipid deposition into the aorta will be vastly facilitated by the presence of fibrin.

Furthermore, the results obtained with the fluorescent antibody technique indicated that accumulations of $\beta$-lipoprotein in the intima were associated with the presence of fibrin. Woolf et al, treated frozen sections of unfixed human aortae with a fluorescein-coupled rabbit anti-human fibrin serum and found that the fluorescent material was concentrated in the same areas as the major collections of fat. These immunohistochemical results are taken as further evidence that the presence of fibrin may be responsible for the deposition of lipid complexes from abnormally lipaemic sera. 


\section{SUMMARY}

(1) Chromatography with citrated ethanol saline system displayed lipids at the origin, only when chromatograms of samples of acid polysaccharides plus plasma were run on filter papers. They were absent from preparations of acid polysaccharides and serum.

(2) Frozen sections of unfixed human aortae were treated with a fluorescein-coupled rabbit anti-human-fibrin serum to identify fibrin. Adjacent sections were treated with a fluorescent-coupled rabbit anti-human- $\beta$-lipoprotein serum. A striking feature throughout is the constancy with which the fluorescein-labelled anti-fibrin serum was concentrated in the same areas as the major collections of the fluorescent-coupled anti- $\beta$-lipoprotein serum.

It is suggested that fibrinogen enhances the deposition of lipid complexes from abnormally lipaemic sera.

\section{REFERENGES}

1. Murakami, M., et al. : Jap. Heart J. 3: 563, 1962.

2. Yasuda, Y.: Jap. Circulat. J. $27: 519,1963$.

3. Bernfeld, P., Donahue, V. M., and Berkowitz, M.E. : J. Biol. Chem. 51 : 226, 1957.

4. Dyrbye, M. and Kirk, J. E. : J. Geront. 12: 20, 1957.

5. Crawford, T. and Woolf, N. : J. Path. Bact. $79: 221,1960$.

6. Woolf, N. and Crawford, T.: J. Path. Bact. 80: 405, 1960.

7. Stewart, G. T. : Brit. J. Exp. Path. 43: 345, 1962. 